\title{
The question of bidirectional associations in pigeons' learning of conditional discrimination tasks
}

\author{
RALPH W. RICHARDS \\ Colorado State University, Fort Collins, Colorado
}

\begin{abstract}
Three transfer-of-learning experiments were conducted to determine whether the associations or rules learned by pigeons in a conditional discrimination are bidirectional. Statistically significant evidence for bidirectionality was obtained in two of the experiments.
\end{abstract}

In a conditional discrimination, responding to one stimulus is correct or incorrect depending on the value of some other stimulus. Consider a three-key symbolic matching-to-sample task with pigeons in a Skinner box. On any given trial, sample stimulus A or B is briefly presented on the center key, followed by comparison stimuli $\mathrm{X}$ and $\mathrm{Y}$ on the side keys. Choice of $\mathrm{X}$ is correct after $\mathrm{A}$, and choice of $\mathrm{Y}$ is correct after B. Hogan and Zentall (1977) used this task to examine whether the rules or associations that pigeons learn are bidirectional. Specifically, they used a transfer-of-learning paradigm to determine whether pigeons that received the aforementioned training would more quickly learn a task that rewarded them for choosing $\mathrm{A}$ after $\mathrm{X}$ and $\mathrm{B}$ after $\mathrm{Y}$ than a task that rewarded them for choosing $B$ after $X$ and $A$ after $Y$. There was little or no difference in the speed with which the two transfer tasks were mastered. Gray (1966) and Rodewald (1974) claimed to have found evidence for weak bidirectionality.

The present series of experiments further examined the issue of bidirectional associations in pigeons by using several different conditional discrimination procedures.

\section{EXPERIMENT 1}

Experiment 1 employed a successive symbolic matching task. In the first phase, trials began with either an A or B sample on the center key followed by an X or Y comparison on the same key. Responding to $X$ was reinforced after A, but was not reinforced after B. Conversely, responding to $\mathrm{Y}$ was reinforced after $\mathrm{B}$, but was not reinforced after $A$. In the second phase, $X$ and $Y$ served as samples and $\mathrm{A}$ and $\mathrm{B}$ were comparisons. For pigeons in the congruent $(\mathrm{C})$ group, responding to $\mathrm{A}$ and $\mathrm{B}$ was reinforced after $X$ and $Y$, respectively. For pigeons in the incongruent (I) group, responding to B and A was reinforced after $\mathrm{X}$ and $\mathrm{Y}$, respectively.

Reprints may be obtained from Bill Richards, Department of Psychology, Colorado State University, Fort Collins, CO 80523.

\begin{abstract}
Method
Subjects. Twenty experimentally naive, adult, female White Cameaux pigeons were maintained at $80 \%$ of their free-feeding weights.

Apparatus. Each of the five similar Skinner boxes (approximately $14 \times 14 \times 14$ in.) contained a houselight, food aperture, and three Lehigh Valley Electronics (LVE) keys that could be illuminated by an inline display cell or by LVE tricolor lights. In the present experiment, only the center key and inline display cell were used. Each box was housed in a sound- and light-attenuating chamber and was interfaced to an Apple II computer.

Procedure. After magazine training, shaping, and several sessions of intermittent reinforcement for pecking the white center key, training on the successive matching task was begun. Each session contained 80 trials. The intertrial interval had a minimum duration of $25 \mathrm{sec}$ and ended with the elapse of $5 \mathrm{sec}$ without a peck. Each trial contained, in order, a white warning stimulus (removed after five pecks), a sample stimulus (removed by the first peck after $5 \mathrm{sec}$ ), a darkening of the key for $.5 \mathrm{sec}$, a comparison stimulus, and a 3-sec food reward or blackout. On the positive trials, the reward was produced by the first keypeck occurring at least $5 \mathrm{sec}$ after comparison onset. On negative trials, the blackout automatically occurred $5 \mathrm{sec}$ after comparison onset. Half of the pigeons in each of the two groups received homogeneous red or green lights as samples and three vertical or horizontal white lines on black background as comparisons; the other half received line samples and color comparisons. For all pigeons, during the 65 sessions of Phase 1, the positive trials contained red and vertical or green and horizontal combinations, and the negative trials contained red and horizontal or green and vertical combinations.

During the 25 sessions of Phase 2, pigeons that had received color (line) samples and line (color) comparisons now received line (color) samples and color (line) comparisons. For the 10 pigeons in Group C, the red and vertical or green and horizontal combinations continued to be the positive trials, and the red and horizontal or green and vertical combinations continued to be the negative trials. For the 10 pigeons in Group I, positive trials contained red and horizontal or green and vertical combinations, and negative trials contained red and vertical or green and horizontal combinations.
\end{abstract}

\section{Results}

Accuracy of performance on the matching task was assessed by a discrimination ratio (DR) based on the formula $c /(c+i)$, where $c$ and $i$ represent response rates during the first $5 \mathrm{sec}$ of the correct and incorrect comparisons, respectively. A DR of 1.00 indicates perfect discrimination, and a DR of .50 represents chance performance (i.e., equal response rates to correct and incorrect comparisons).

Discrimination accuracy of both groups gradually improved during Phase 1. The mean DRs based on the last 
five sessions were .84 and $.86[t(18)=.45$, n.s. $]$ for Groups $\mathrm{C}$ and I, respectively. In all statistical analyses, an alpha level of .05 was used. The mean DRs across successive five-session blocks in Phase 2 for the $C$ (I) subjects were $.51(.52), .56(.59), .66(.66), .75(.72)$, and .75 (.76). A two-way analysis of variance (ANOVA) yielded a significant effect for blocks $[F(4,72)=24.66]$, but no significant effects for groups $[F(1,18)=.02]$ or groups $\times$ blocks $[F(4,72)=.33]$. In other words, there was no evidence that pigeons acquired backward associations between sample and comparison stimuli during their learning of the successive symbolic matching task.

\section{EXPERIMENT 2}

Prior to the transfer test in Experiment 1, the pigeons' only experience with A and B was as samples, and this produced rapid nondifferential responding to them. Perhaps the transfer test would have proven more sensitive to backward associations if the pigeons had had some previous experience with $\mathrm{A}$ and $\mathrm{B}$ as comparisons. Accordingly, pigeons in Experiment 2 received training on two matching tasks before the transfer test. On one task $A$ and $B$ were samples and $X$ and $Y$ were comparisons; on the other task A and B became comparisons and two novel stimuli served as samples. The transfer test used $\mathrm{X}$ and $\mathrm{Y}$ samples and $\mathrm{A}$ and $\mathrm{B}$ comparisons. Experiment 2 also attempted to increase the distinctiveness of the sample and comparison portions of a trial by turning the houselight off during the samples and by presenting the samples and comparisons at separate locations.

\section{Method}

After being conditioned to peck the white center key, 20 naive pigeons received, in order, 55 sessions with Task 1,33 sessions with Task 2 , 13 sessions with Task 1 , and 19 sessions in which the tasks alternated daily. For Task 1, all pigeons were rewarded for responding to three vertical lines $(\mathrm{X})$ after a red $(\mathrm{A})$ sample and for responding to a white $\operatorname{dot}(\mathrm{Y})$ after a green (B) sample. Responding to $\mathrm{X}$ and $\mathrm{Y}$ was not reinforced after B and A, respectively. For Task 2 the samples were yellow and blue, the comparisons were red and green, and the rewarded and nonrewarded sequences were counterbalanced. The houselight was illuminated during both sample and comparison stimuli in the first $\mathbf{7 5}$ sessions, but was not illuminated during the sample stimuli in later sessions. Samples always appeared on the right key, and comparisons always appeared on the center key.

During the next 18 sessions, Task 1 alternated daily with the transfer task. For the final 30 sessions, a single session with Task 1 was inserted between every 5 sessions with the transfer task. On the transfer task, subjects in Group C were rewarded only for responding to A after X and for responding to B after Y. Subjects in Group I were rewarded only for responding to $A$ after $Y$ and $B$ after $X$.

Delay between sample offset and comparison onset was $.5 \mathrm{sec}$ during Sessions 1-40 and 56-75 and was $1 \mathrm{sec}$ during sessions 41-55; thereafter, no delay occurred between sample and comparison.

\section{Results}

The mean DRs based on the last five sessions of Task 1 before the transfer test were .90 and .91 for Groups $\mathrm{C}$ and $I$, respectively $[t(18)=.38$, n.s.]. On Task 2 the mean DRs were .75 and $.76[t(18)=.12$, n.s. $]$.
During successive blocks of the transfer task, the mean DRs for Group C (I) were .50 (.50), .51 (.50), .53 (.52), $.60(.53), .63(.53), .66(.55)$, and $.71(.58)$. The ANOVA yielded significant effects for blocks $[F(6,108)=8.96]$ and for the groups $\times$ blocks interaction $[F(6,108)=$ 2.27]. The significant interaction suggests that some backward association was formed. The groups effect was not significant $[F(1,18)=2.52]$.

\section{EXPERIMENT 3}

In the previous experiments, two stimuli were presented successively, and whether responding to the second was reinforced depended on the value of the first. In other words, not only did the second stimulus have a signal function, but the level of responding to it also served as the index of discrimination. Perhaps this dual aspect of the second stimulus hindered the development or detection of backward associations. Such associations might have been more apparent if the signal and response-indexing functions of the second stimulus were separated.

Toward this end, Experiment 3 employed a different conditional discrimination task. As before, each trial contained successive presentation of two stimuli on the center key, but their values determined whether subsequent choice of the left or the right key was reinforced. On the initial task, choice of the left-side key was reinforced after $\mathrm{A}-\mathrm{X}$ or $\mathrm{B}-\mathrm{Y}$ sequences and choice of the right-side key was reinforced after A-Y or B-X sequences. On the transfer test, pigeons in Group $C$ were rewarded for pecking left after $\mathrm{X}-\mathrm{A}$ and $\mathrm{Y}$-B sequences and were rewarded for pecking right after $\mathrm{Y}-\mathrm{A}$ or $\mathrm{X}-\mathrm{B}$ sequences. Pigeons in Group I received the converse contingencies on the transfer test.

\section{Method}

After being trained to keypeck, the 20 naive pigeons received 85 sessions with Task 1. For each trial, two 5 -sec stimuli were projected on the center key, followed immediately by illumination of the side keys with identical orange lights. The first center-key stimulus could be homogeneous red (A) or blue (B) lights, and the second stimulus could be a white triangle $(\mathrm{X})$ or vertical lines $(\mathrm{Y})$ on black backgrounds. Choice of the left-side key was reinforced after $A-X$ and $B-Y$ sequences, and choice of the right-side key was reinforced after $A-Y$ and $B-X$ sequences. $A$ correction procedure was used, and sessions contained 40 reinforced trials.

During the next 45 sessions, Task 1 occurred on Sessions 2, 4, 6, $8,19,20$, and 31-35; Task 2 appeared on the remaining sessions. For Task 2, the illumination of the center key began with $X$ or $Y$ and ended with A or B. Pigeons in Group C (I) were rewarded for choosing the left (right) key after $\mathrm{X}-\mathrm{A}$ and $\mathrm{Y}-\mathrm{B}$ sequences and for choosing the right (left) key after $X-B$ and $Y-A$ sequences.

\section{Results}

The mean percentage of correct choices (excluding correction trials) during the last block of Phase 1 was $83 \%$ and $82 \%$ for Group C and Group I, respectively. During successive blocks of Task 2, which was terminated prematurely because lack of funds prevented continued feeding and adequate care for the pigeons, the mean percentages 
of correct choices for Group C (I) were $52 \%$ (48\%), $53 \%$ (48\%), 55\% (51\%), 54\% (48\%), 57\% (51\%), 56\% (51\%), and $57 \%(51 \%)$. Birds in Group C showed slightly (4\%-6\%) better performance than birds in Group I, and an ANOVA on Task 2 revealed significant effects for groups $[F(1,18)=7.37]$ and blocks $[F(6,108)=3.54]$ but not for the groups $\times$ blocks interaction $[F(6,108)=$ .10].

\section{GENERAL DISCUSSION}

The present experiments employed a transfer-of-learning paradigm to determine whether the associations or rules learned by pigeons in a conditional discrimination task are bidirectional. Pigeons first mastered a discrimination containing four trial types composed of one of two stimuli (A or B) followed by one of two different stimuli (X or Y). The value of the first stimulus in conjunction with the value of the second stimulus signaled whether or not responding to the second stimulus would be reinforced (Experiments 1 and 2) or which side key should be chosen to obtain reward (Experiment 3). The transfer task reversed the order of the successively presented stimuli ( $\mathrm{X}$ or $\mathrm{Y}$ was followed by $\mathrm{A}$ or $\mathrm{B}$ ). For half of the subjects, the same combinations of stimuli were used to indicate whether responding to the second stimulus would be reinforced (Experiments 1 and 2) or to indicate which side key should be chosen (Experiment 3). For the other half, the other combination signaled whether subjects should respond to the second stimulus or choose the left- or right-side key. Bidirectionality was assessed by comparing the performance of the two groups on the transfer task.

In Experiment 1 all stimuli were presented on the same key, and the pigeons' first experience with A and B in the second position occurred during the actual transfer task. There was no evidence of bidirectionality. Experiment 2 increased the distinctiveness of the sample and comparison portions of each trial (by projecting the two stimuli on different keys and by having them accompanied by different conditions of houselight illumination) and provided prior conditional discrimination ex- perience with the $A$ and $B$ in the second position before the transfer task. A statistically significant amount of bidirectionality was obtained; however, the magnitude was not large. Experiment 3 required subjects to make differential choice responding on the side keys, and a small, but significant, amount of bidirectionality was again obtained. Overall, the present results are in accord with prior reports (Gray, 1966; Hogan \& Zentall, 1977; Rodewald, 1974) in showing no more than weak bidirectionality in the pigeon. However, the paucity of systematic research would make it premature to conclude that pigeons never evidence strong bidirectionality. Stronger bidirectionality may occur if selective aspects of the present methodologies are combined with several other procedural variations. For example, it might be useful to (1) present each of the two successive stimuli ( $A$ or $B$ and $X$ or $Y$ ) at different locations or under different houselight illumination conditions, (2) require a choice after presentation of the two successive stimuli, (3) permit this choice to occur immediately after the second stimulus is shown, (4) provide other conditional discrimination experiences with $X$ and $Y$ appearing first and with A and B appearing second, (5) randomly intermix all of these conditional discrimination trials during training and transfer testing, and/or (6) include some conditional discriminations in which the sample stimuli repeatedly alternate back and forth (see Richards \& Bowers, 1985).

\section{REFERENCES}

Gray, L. (1966). Backward association in pigeons. Psychonomic Science, 4, 333-334.

Hogan, D. E., \& Zntall, T. R. (1977). Backward associations in the pigeon. American Journal of Psychology, 90, 3-15.

RicharDS, R. W., Bowers, R. L. (1985). Dual-element effects in a one-key matching task with simultaneous and sequential elements. Psychological Record, 35, 575-582.

Rodewald, H. K. (1974). Symbolic matching-to-sample by pigeons. Psychological Reports, 34, 987-990.

(Manuscript received March 30, 1988.) 